УДК 519.1

\title{
Interval Estimation of a System Balance Based on the Conflict Theory
}

\author{
Valery V.Menshikh* \\ Oleg V. Pyankov ${ }^{\dagger}$ \\ Voronezh Institute of the Ministry of the Interior \\ Patriotov, 53, Voronezh, 394065 \\ Russia
}

Received 01.12.2016, received in revised form 10.06.2017, accepted 20.03.2018

The possibility of a study of systems based on the conflict theory is examined in this article. Interval estimates of a system balance, which consider the conflicting properties of its elements, are suggested. The integrated assessment approach is developed for a complex system in view of time and weight parameters. A numerical example of its use is presented for analyzing of agraph model of an information-analytical system.

Keywords: balance, interval estimates of the conflict, information-analytical systems.

DOI: 10.17516/1997-1397-2018-11-2-249-257.

\section{Introduction}

It is now well-established from a variety of studies that system representation of modeled objects usually involves their description as a final set of elements and mutual relations among them. Among the indicators of efficiency, which show the effectiveness of such systems' performance, the significant place is reserved to their balance, i.e. an absence of internal conflicts and compromises. Subject areas, within which a system balance is traditionally examined, include psychology (persistence of small groups), biology (food pyramid), economics (transport networks and urban economy), computer systems [1-3]. The presence of a wide variety of elements and relations between them in these areas allows us to refer them to complex systems. Norkin et al. presents kinds of uncertainty, arising in an analysis and evaluation of complex systems [4]. Stages of research systems are integral to the design of information systems and have a number of specific features. This raises problems, associated with resolution of conflicts between its subsystems.

We can determine two ways of a complex studying for a conflict system:

1. A description of elements' interaction in general, considering all relevant factors, detection and investigation of conflicting parties, possible nature of their interaction, causes and mechanisms of a conflict.

2. With the assumption that a cause and nature of a conflict are known to Parties, the selection of the main, from the researcher's point of view, factor (as maximum: of 2-3 factors) and construction of a model for assessing of a significance of the priori factors and results of a conflict.

\footnotetext{
*menshikh@mail.ru

†pyankovov@vimvd.ru

(c) Siberian Federal University. All rights reserved
} 
Some authors [5-7] examined such concepts as a degree and intensity of a conflict, as well as ways of their measurement. Sysoev considered a structure-parametric interaction of subsystems [8]. The area of conflict interactions and particularities of a choice of decisions is formalized in them. Levin found that the relations of conflict, cooperation and neutrality between systems, described by the algebra of logic, could be successfully explored using the same mathematical apparatus [9]. This enables to use constructive approach to the study, efficiency of computing algorithms and ease in interpreting results.

Now it is possible to talk about a compromise solution to a conflict, associated with selection and development of different compromise schemes, and about optimization, related to search of the introduced terms. At the same time, assessments of a system balance were provided in general terms, i.e. they were not based on and, accordingly, did not take into account the conflicting properties of individual elements. Taking into account of a conflict of individual elements will give an opportunity to develop a more careful approach to a system balance analysis and will provide effective measures to improve it. Determination of relations, prevailing over the other ones in a system, allows to draw conclusions on effectiveness of its functioning and to make recommendations on application of certain control actions.

\section{Formulation of the problem.}

To describe relationships between the elements, we use the terminology of the conflict theory [7].

We will consider the two elements $S_{1}, S_{2}$ which form some system $S$ with the general purpose $W$. In achieving this purpose, subsystems interact in accordance with their local purposes $W_{1}, W_{2}$. We introduce the following set

$$
S=\left\{S^{1}=\left(S_{1}, S_{2}\right), S^{2}=\left(S_{1}, \overline{S_{2}}\right), S^{3}=\left(\overline{S_{1}}, S_{2}\right)\right\},
$$

where $\overline{S_{i}}$ is an absence of the subsystem $S_{i}$ in the environment $S$.

We assume that there are real functions (for example, the utility function) $q(S)$ such as if $S_{1}, S_{2} \subset S$ and $S_{1} \succ^{W} S_{2}\left(\succ^{W}\right.$ is better in the sense of $W$ ), then

$$
q\left(S_{1}\right)>q\left(S_{2}\right) .
$$

We assume that the system $S_{2}$ conflicts with the system $S_{1}$ in the sense of achieving $W\left(S_{2}>I S_{1}\right)$, if

$$
q\left(S^{1}\right)=q\left(S_{1}, S_{2}\right)<q\left(S_{1}, \overline{S_{2}}\right)=q\left(S^{2}\right) .
$$

It means that presence of $S_{2}$ in the $S$ system's environment reduces the overall system's utility in achieving the purpose $W$ in accordance with the criterion $q$.

Without examining issues, related to definition of q, we assume that there are no contradictions in the methods of its calculation [10].

It is obvious to assume that there are other relationships in this case, providing fulfillment of the conditions $S^{1}$ and $S^{2}$ :

$$
\begin{aligned}
& q\left(S^{1}\right)=q\left(S_{1}, S_{2}\right)>q\left(S_{1}, \overline{S_{2}}\right)=q\left(S^{2}\right), \\
& q\left(S^{1}\right)=q\left(S_{1}, S_{2}\right)=q\left(S_{1}, \overline{S_{2}}\right)=q\left(S^{2}\right) .
\end{aligned}
$$


In (2), a presence of $S_{2}$ increases the expected utility in the sense of the purpose $W$, and it is said that $S_{2}$ cooperates with $S_{1}\left(S_{2}>I_{c} S_{1}\right)$. If a presence of $S_{2}$ does not affect the overall utility (3), $S_{2}$ and $S_{1}$ are independent $\left(S_{2}>I_{i} S_{1}\right)$.

Biology is a good illustration of those relationships. For example, $S_{1}$ means a grass, $S_{2}$ is a sheep, and $S_{3}$ is wolves. Therefore, we can write that:

- dense grass is favorable for sheep $S_{1}>I_{c} S_{2}$ and does not influence (directly) on wolves $S_{1}>I_{i} S_{3}$

- increase in quantity of sheep is good for wolves $S_{2}>I_{c} S_{3}$, but bad for a grass $S_{2}>I S_{1}$;

- increase in quantity of wolves is bad for a sheep $S_{3}>I S_{2}$, but does not influence (directly) to a grass $S_{3}>I_{i} S_{1}$.

To study the structural characteristics of the conflict relationships of any system, a directed graph, all arcs of which are assigned the "+" or "-" signs, can be used. Let indicate an orgraph as

$$
G=G(S, U),
$$

where $S$ is a set of vertices of the orgraph, corresponding to the system elements, $|S|$ is cardinality; $U$ is a set of the or graph's arcs, corresponding to binary relations between the system elements.

We attribute the " + " and "-" signs to each arc as follows:

- if $S_{i}$ conflicts with $S_{j}$, then the arc $u_{i j}$ is provided with the "-" sign;

- if $S_{i}$ cooperates with $S_{j}$, then the arc $u_{i j}$ is provided with the "+" sign;

- if $S_{i}$ and $S_{j}$ are independent elements, the arc $u_{i j}$ is not depicted.

We call a cycle as balanced, if the number of arcs $u_{i}$ with the "-" sign is even. Accordingly, the graph is balanced, if all cycles of the graph are balanced.

The theoretical analysis of a balance at the level of structural analysis is carried out by means of assessments, imposed by Harari [1]:

$$
\begin{aligned}
& M^{-}=C^{-} / C=\left(C-C^{+}\right) / C, \\
& M^{+}=C^{+} / C=\left(C-C^{-}\right) / C,
\end{aligned}
$$

where $C$ is the number of all directed cycles (hereinafter - dicycles), $C^{+}$is the number of balanced dicycles, $C^{-}$is the number of unbalanced dicycles.

However, the non sufficiency of a graph's balance assessment (conflict of the system elements) can be assumed only through the ratio of balanced and unbalanced cycles.

\section{Decision}

\subsection{Definitions}

To study the graphs' balance, we introduce the following definitions:

Def. 1. The graphs' node $s_{i}^{c}$ is considered as cycle-balanced, if all cycles, to which it belongs, are balanced.

Def. 2. The graphs' node $s_{i}^{s}$ is considered as system-balanced,if it does not belong to any of the cycles. 
Def. 3. The graphs' node $s_{i}^{n}$ is considered as unbalanced, if at least one of the cycles, to which it belongs, is unbalanced.

It should be noted that after determining the type of all graphs' nodes, the following condition should be met:

$$
\left|s^{c}\right|+\left|s^{s}\right|+\left|s^{n}\right|=|S|,
$$

where $\left|s^{c}\right|$ is cardinality of the set of cycle-balanced nodes; $\left|s^{n}\right|$ is cardinality of the set of unbalanced nodes; $\left|s^{s}\right|$ is cardinality of the set of system-balanced nodes.

We introduce the definitions, by use of which we measure the dynamic properties of conflict elements in a system.

Def. 4. The graphs' node $s_{i}^{D k}$ is considered as $k$-th degree dynamic, if a length of the shortest cycle, which includes this node, is equal to $k$.

Def. 5. The graphs' node $s_{i}^{S k}$ is considered as $k$-th degree static, if a length of the longest cycle, which includes this node, is equal to $k$.

The system elements interact with each other differently, i.e. this interaction can be strong and tangible between some of them, and it can be weak among some others, so we can neglect it as a result. In connection with this, the problem of development of a system model, based on a "force" of interaction, arises. Introducing of a weight of the cycle, to which a node belongs, allows analyzing of or graph's cycles by importance and its influence on parameters of the system elements. The analysis of the cycles' weight allows to reveal cycles with minimum $V_{\text {min }}$ and maximum $V_{\max }$ weight and to introduce the following definitions:

Def. 6. The graphs' node $s_{i}^{V_{\max }}$ is considered as $V_{\max }$-th degree strong, if the maximum weight of a cycle, which the node belongs to, is equal to $V_{\max }$.

Def. 7. The graphs' node $s_{i}^{V_{\min }}$ is considered as $V_{m i n}$-th degree weak, if the minimum weight of a cycle, which the node belongs to, is equal to $V_{\min }$.

In the above-mentioned estimation methods of influenceof one element on another, the issue of thetransfer between the system elements was not touched upon.

Supposing that at the initial time the value of the utility function $S_{i}$ is $q_{i}^{0}, S_{i}$ affects another element $S_{j}$, the utility function value of which is $q_{j}^{0}$. Assume that for some reason the parameter value $S_{i}$ for the time interval $t_{1}$ is changed to a value $q_{i}^{1}$ that results in the transmission of this change to the element $S_{j}$ during $t_{2}$. There is a change in the parameter value of the element $S_{j}$ to a value $q_{j}^{1}$ that takes the time interval $t_{3}$. Therefore, the weight of the arc in the or graph's model, taking into account the time interval, is equal to the time interval $t_{2}$, and the whole process of changing of parameter values of the system elements will take time, equal to

$$
T=t_{1}+t_{2}+t_{3} .
$$

Depending on nature of the tasks, the values $t_{i}$ of expression (7) can assume the value of zero. To investigate the cyclic properties of the system, the expression (7) can easily be converted into

$$
T_{\text {cycle }}=t_{1}+t_{2}+t_{3}+t_{4}+t_{5}
$$

where $t_{4}$ is the time, which is necessary for transfer of changes from the element $S_{j}$ to the element $S_{i} ; t_{5}$ is the time, which is necessary for change of a parameter value of the element $S_{i}$ from $q_{i}^{1}$ to $q_{i}^{2}$.

Applying increment $\Delta t$ allows to convert the expressions (7) and (8) into the following form:

$$
T=a_{1} \Delta t+a_{2} \Delta t+a_{3} \Delta t=\sum a_{i} \cdot \Delta t=N \cdot \Delta t
$$




$$
T_{\text {cycle }}=a_{1} \Delta t+a_{2} \Delta t+a_{3} \Delta t+a_{4} \Delta t+a_{5} \Delta t=\sum a_{i} \cdot \Delta t=N_{\text {cycle }} \cdot \Delta t,
$$

Then, we can introduce the following definitions to analyze the elements of the system, represented by an or graph:

Def. 8. The graphs' node $s_{i}^{B k}$ is considered as $k$-th degree fast, if the minimum $T_{\text {cycle }}$ of all cycles, which include the node, is equal to $k \cdot \Delta t$.

Def. 9. The graphs' node $s_{i}^{M k}$ is considered as $k$-th degree slow, if the maximum $T_{c y c l e}$ of all cycles, which include the node, is equal to $k \cdot \Delta t$.

\subsection{Estimation of a balance}

The definitions 1-9 make possible an assessment of a conflict system through its elements.

Since a node can enter into balanced and unbalanced cyclessimultaneously, the following assessments of the vertices' balance can be introduced:

1. Estimation of a node's balance by cycles:

$$
M_{i}^{+}=C^{+}\left(s_{i}\right) / C\left(s_{i}\right)
$$

where $C\left(s_{i}\right)=C^{+}\left(s_{i}\right)+C^{-}\left(s_{i}\right)$ is the total number of cycles, to which the node $s_{i}$ belongs. If $M_{i}^{+}=1$, the node $s_{i}$ is considered as balanced.

2. Estimation of a node's imbalance by cycles:

$$
M_{i}^{-}=C^{-}\left(s_{i}\right) / C\left(s_{i}\right) .
$$

If $M_{i}^{-}=1$, the node $s_{i}$ is considered as unbalanced.

3. Estimation of a graph's balance, based on its nodes:

$$
M_{c}=\left|s^{c}\right| /|S| \text {. }
$$

4. Estimation of a graph's balance by system:

$$
M_{s}=\left|s^{s}\right| /|S|
$$

5. Estimation of a graph's imbalances:

$$
M_{n}=\left|s^{n}\right| /|S|
$$

6. Estimation of the average balance and imbalance of a graph:

$$
M_{m}^{+}=\frac{1}{|S|} \sum_{i=1}^{|S|} M_{i}^{+}, \quad M_{m}^{-}=\frac{1}{|S|} \sum_{i=1}^{|S|} M_{i}^{-} .
$$

7. Estimation of a graph's dynamics:

$$
M_{d k}=\left|s^{D k}\right| /|S| .
$$

8. Estimation of a graph's statics:

$$
M_{s k}=\left|s^{S k}\right| /|S| .
$$




\subsection{Interval estimation}

The proposed estimation indicators have the point values, which makes the analysis difficult. In this regard, it may be suggested to use interval estimates, which consist in choosing of some limit values $M k_{\text {min level }}, V_{\text {max level }}$ etc., where sets of slow, heavy elements are formed, instead of point values. In other words, these sets will include only those elements, whose parameter values exceed the boundary in the case of calculating the maximum estimates or do not exceed it in the case of calculating the minimum estimates. In general, the subject area and nature of the research should determine the choice of limit values. Then, respectively, for interval evaluations, the expressions(16) and (17) take the following form:

1. Interval K-degree system dynamics

$$
M_{d k}=\left|s^{D_{k l e v}}\right| /|S|,
$$

where $s^{\text {Dk lev. }}$ is the set of graphs' nodes, for which $D_{k} \leqslant D_{k l e v}$.

2. Interval K-degree system statics

$$
M_{s k}=\left|s^{S k l e v} \cdot\right| /|S|,
$$

where $s^{S k l e v}$. is the set of graphs' nodes, for which $S_{k} \geqslant S_{k}$ lev.

3. Interval estimation of strong elements of the system:

$$
M_{V_{\max }}=\left|s^{V_{\max l e v}} \cdot\right| /|S|,
$$

where $s^{V_{\max } \text { lev }}$ is the set of system elements, for which the $V_{\max } \geqslant V_{\max }$ lev.

4. Interval estimation of weak elements of the system:

$$
M_{V_{\min }}=\left|s^{V_{\text {min lev }}}\right| /|S|,
$$

where $s^{V_{\text {min lev }}}$ is the set of system elements, for which $V_{\min } \leqslant V_{\min }$ lev.

5. Interval estimation of fast elements of the system:

$$
M_{B}=\left|s^{B_{\text {kmaxlev }}}\right| /|S|,
$$

where $s^{B_{\text {kmaxlev }}}$ is the set of system elements, for which $B_{k \max } \geqslant B_{k \text { maxlev. }}$.

6. Interval estimation of slow elements of the system:

$$
M_{M}=\left|s^{M_{k m i n l e v}}\right||/ S|,
$$

where $s^{M_{k m i n l e v}}$ is the set of system elements, for which $M_{k m i n} \leqslant M_{k m i n l e v}$.

\section{A numerical example}

In [12], the balance estimates for aninformation-analytical system's model, represented as an or graph $G_{W a r}$, are provided (Tab. 1). A calculation of the orgraph $G_{W}$ 's balance estimates for two changes of the structural-parametric model (Tabs. 2 and 3), is also given in this work. Let's carry out a calculation of interval balance estimates and analyze the advantages of their use (Tab. 4).

Despite the fact that the values did not change for three interval estimates $\left(M_{d k}, M_{V_{\max }}\right.$, $M_{B}$ ) during the structural transformation of the graph $G_{W}$, the following conclusions can be drawn, which are applicable to the studied system: 
- for $91 \%$ of all elements, the shortest cycle, in which it is located, is a cycle with length, equal to 2, i.e., in fact, most of the elements have the pairwise interaction;

- most elements $(82 \%)$ are in long cycles, indicating the high connectivity of the system. Adding of an arc in this example increases the connectivity of the system up to $91 \%$, thereby changing one element affects the majority of other elements of the system;

- $73 \%$ of the elements belong to strong cycles, so small changes of an element's utility may lead to volatility in the other elements' usefulness;

- $64 \%$ of the elements are in weak interactions, where changes of one element do not greatly affect other elements. It can be suggested that removal of strong cycles will not lead to the system stability, but it is necessary for account of weak effects when analyzing the system. Adding of a cooperative relationship leads to an increase in the percentage (73\%) of weak interactions. In this sense, it becomes interesting to find the strength factor, which determines the ratio of strong and weak cycles in the system:

$$
K_{V}=M_{V_{\max }} / M_{V_{\min }}, \text { at } V_{\text {max lev. }}=V_{\text {minlev. }}
$$

- $82 \%$ of the system elements are in fast cycles, allowing to draw a conclusion about the possibility of a high speed modifications' transfer within the system;

- $91 \%$ of the system elements are in slow cycles, so the aftereffect of one system element's change is still long enough to pass through the system before return to the source. Introducingof an additional arc increases the percentage up to $100 \%$ and removal of an arc leads to the decrease to $82 \%$.

Table 1. The values of balance estimates for the graph $G_{W}$

\begin{tabular}{lccccccccccc}
\hline Nodes & $s_{1}$ & $s_{2}$ & $s_{3}$ & $s_{4}$ & $s_{5}$ & $s_{6}$ & $s_{7}$ & $s_{8}$ & $s_{9}$ & $s_{10}$ & $s_{11}$ \\
\hline$M^{+}$ & 0.57 & 0.4 & 0.14 & 0.63 & 1 & 0.83 & 0.75 & 0.6 & 0.68 & 0.67 & 0.71 \\
$D_{k}$ & 2 & 2 & 2 & 2 & 2 & 2 & 2 & 4 & 2 & 2 & 2 \\
$S_{k}$ & 9 & 9 & 9 & 9 & 2 & 9 & 9 & 6 & 9 & 9 & 9 \\
$V_{\max }$ & 1 & 1 & 0.32 & 0.8 & 0.3 & 1 & 1 & 0.3 & 0.8 & 1 & 1 \\
$V_{\min }$ & 0.077 & 0.077 & 0.077 & 0.022 & 0.3 & 0.072 & 0.022 & 0.022 & 0.022 & 0.022 & 0.022 \\
$B_{k}$ & 3 & 3 & 12 & 2 & 5 & 3 & 2 & 7 & 3 & 2 & 2 \\
$M_{k}$ & 23 & 23 & 23 & 23 & 5 & 23 & 23 & 22 & 23 & 23 & 23 \\
\hline
\end{tabular}

Table 2. The values of balance estimates for the graph $G_{W}$, when adding a cooperative relationship $S_{11}>I_{c} S_{5}$

\begin{tabular}{lccccccccccc}
\hline Nodes & $s_{1}$ & $s_{2}$ & $s_{3}$ & $s_{4}$ & $s_{5}$ & $s_{6}$ & $s_{7}$ & $s_{8}$ & $s_{9}$ & $s_{10}$ & $s_{11}$ \\
\hline$M^{+}$ & 0.57 & 0.4 & 0.14 & 0.57 & 0.2 & 0.62 & 0.65 & 0.6 & 0.59 & 0.57 & 0.62 \\
$D_{k}$ & 2 & 2 & 2 & 2 & 2 & 2 & 2 & 4 & 2 & 2 & 2 \\
$S_{k}$ & 9 & 9 & 9 & 7 & 9 & 9 & 9 & 6 & 9 & 9 & 9 \\
$V_{\max }$ & 1 & 1 & 0.32 & 0.8 & 1 & 1 & 1 & 0.3 & 0.8 & 1 & 1 \\
$V_{\min }$ & 0.077 & 0.077 & 0.077 & 0.022 & 0.72 & 0.072 & 0.022 & 0.022 & 0.022 & 0.022 & 0.022 \\
$B_{k}$ & 3 & 3 & 12 & 2 & 5 & 3 & 2 & 7 & 3 & 2 & 2 \\
$M_{k}$ & 23 & 23 & 23 & 23 & 21 & 23 & 23 & 22 & 23 & 23 & 23 \\
\hline
\end{tabular}


Table 3. The values of balance estimates for the graph $G_{W}$, when eliminating an impact of the conflict $S_{8}>I S_{9}$

\begin{tabular}{lccccccccccc}
\hline Nodes & $s_{1}$ & $s_{2}$ & $s_{3}$ & $s_{4}$ & $s_{5}$ & $s_{6}$ & $s_{7}$ & $s_{8}$ & $s_{9}$ & $s_{10}$ & $s_{11}$ \\
\hline$M^{+}$ & 0.57 & 0.4 & 0.14 & 0.67 & 1 & 0.83 & 0.79 & 1 & 0.76 & 0.73 & 0.77 \\
$D_{k}$ & 2 & 2 & 2 & 2 & 2 & 2 & 2 & 4 & 2 & 2 & 2 \\
$S_{k}$ & 9 & 9 & 9 & 9 & 2 & 9 & 9 & 6 & 9 & 9 & 9 \\
$V_{\max }$ & 1 & 1 & 0.32 & 0.8 & 0.3 & 1 & 1 & 0.3 & 0.8 & 1 & 1 \\
$V_{\min }$ & 0.077 & 0.077 & 0.077 & 0.022 & 0.3 & 0.072 & 0.022 & 0.022 & 0.022 & 0.022 & 0.022 \\
$B_{k}$ & 3 & 3 & 12 & 2 & 5 & 3 & 2 & 7 & 3 & 2 & 2 \\
$M_{k}$ & 23 & 23 & 23 & 23 & 5 & 23 & 23 & 14 & 23 & 23 & 23 \\
\hline
\end{tabular}

Table 4. The values of interval balance estimates for the considered states of the model $G_{W}$

\begin{tabular}{lccc}
\hline Interval estimation & The initial state $G_{W}$ & $G_{W^{+}}\left(S_{11}>I_{c} S_{5}\right)$ & $G_{W^{-}}\left(S_{8}>I S_{9}\right)$ \\
\hline$M_{d k}$, at $D_{k} l e v=2$ & 0.91 & 0.91 & 0.91 \\
$M_{s k}$, at $S_{k} l e v=7$ & 0.82 & 0.91 & 0.82 \\
$M_{V_{\text {max }}}$, at $V_{\text {max lev }}=0.8$ & 0.73 & 0.73 & 0.73 \\
$M_{V_{\text {min }}}$ at $V_{\text {min lev }}=0.072$ & 0.64 & 0.73 & 0.64 \\
$M_{B}$ at $B_{\text {kmaxlev }}=5$ & 0.82 & 0.82 & 0.82 \\
$M_{M}$, at $M_{\text {kminlev }}$ & 0.91 & 1 & 0.82 \\
\hline
\end{tabular}

\section{Conclusion}

The study of complex systems in order to identify contradictions in them and to prepare rational solutions for their reduction or elimination requires a thorough analysis of the important and significant interactions between elements of the system. The approach for solving of this problem based on the conflict theory and estimates, givenin the article,allowto obtain not only qualitative, but also quantitative characteristics of a balance of the whole system and its elements. The use of interval estimates provides more accurate balance analysis of complex systems and their components. The detection of conflicting elements can be used for structural changes in the system and it would be a value creating area for further work.

\section{References}

[1] S.Roberts, Fred, Mathematical models with application to social, biological and enviromental problems, Englewood Cliffs, 1986.

[2] G.P.Mojarov, Conflict Resolution in Computer Systems Science and Education of the Bauman MSTU, (2015), no. 8, 184-194 (in Russian).

[3] G.A.Ostapenko, D.G.Plotnikov, Yu.N.Guzev, Formalization of the description of the network conflict, Informatsia \& Bezopasnost', 19(2016), 232-37 (in Russian).

[4] O.R.Norkin, S.S.Parfenova, Conflicts in the design of information systems as a result of uncertainty factor, Journal Sib. Fed. Univ. Enginer. Nauki, 6(2014), 164-67 (in Russian). 
[5] V.V.Druzhinin, D.S.Kontorov, M.D.Kontorov, Introduction to the theory of conflict, Moscow, Radio i svjaz, 1989 (in Russian).

[6] T.L.Saati, Mathematical models of arms control and disarmament, John Wiley \& Sons, 1968 (in Russian).

[7] V.V.Sysoev, Ju.S.Serbulov, R.A.Soloduha, Simulation of discrete measuring information systems, situational management in their structural and parametric representation, Voronezh. Gos. Tehnolog. Akademiya, 2003 (in Russian).

[8] D.V.Sysoev, Conditions of formation of the conflict in the given systems, Information technologies in construction, social and economic systems, (2013), no, 1, 41-48 (in Russian).

[9] V.I.Levin, Elements of the logic-algebraic theory of conflict, Vestnik Lobachevskii Gos. Univer., Nizhni Novgorod, 5(2012), no. 2, 138-147 (in Russian).

[10] V.V.Velichko, Conflicts between the methods of mathematics and mechanics and technological disasters Intelligent systems, Theory and applications. Series "Computer science and applied research", 17(2013), no. 1-4, 427-70 (in Russian).

[11] V.A.Svetlov, Introduction to unified theory of Conflict Analysis and Resolution, Moscow, Librokom, 2012 (in Russian).

[12] V.V.Menshikh, O.V.Pyankov, Structural Parametric Modelling of an Information-Analytical System, Bulletin of the South Ural State University. Series "Mathemathical modeling, Programming \& Computer Software", 9(2016), no. 1, 105-13 (in Russian).

\section{Интервальные оценки сбалансированности системы на основе теории конфликтов}

\section{Валерий В. Меньших \\ Олег В. Пьянков}

Воронежский институт МВД России

Патриотов, 53, Воронеж, 394065

Россия

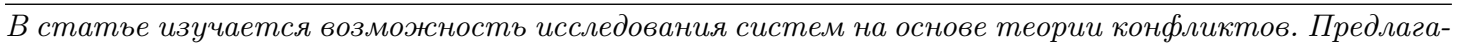
ются интервалъные оценки равновесия системы, которые учитывают конфликтующие свойства ее элементов. Развивается интегралъный подход к очениванию сложной системы с точки зрения временных и весовых параметров. Представляется численный пример анализа графовой модели информационно-аналитической системы.

Ключевые слова: сбалансированность, интервальные оценки конфликта, информачионно-аналитические системы. 